\title{
Fermionic decays of sfermions in the MSSM: a full one-loop calculation *
}

\author{
$\underline{\text { Jaume Guasch }}^{\mathrm{a}}$, Wolfgang Hollik ${ }^{\mathrm{b}}$, Joan Solàc \\ aTheory Group LTP, Paul Scherrer Institut, CH-5232 Villigen PSI, Switzerland. \\ bMax-Planck-Institut für Physik, Föhringer Ring 6, D-80805 München, Germany. \\ cDepartament d'Estructura i Constituents de la Matèria, Universitat de Barcelona, Diagonal 647, \\ E-08028 Barcelona, Catalonia, Spain, and Institut de Física d'Altes Energies, Universitat Autònoma de \\ Barcelona, E-08193 Bellaterra., Barcelona, Catalonia, Spain.
}

We present a full one-loop calculation of the electroweak corrections to the partial decay widths of the fermionic modes of sfermions $\Gamma\left(\tilde{f} \rightarrow f^{\prime} \chi\right)$. The main technical points of the renormalization are presented, and the main features of the results are discussed.

PSI-PR-02-11, UB-ECM-PF-02-20, hep-ph/0210118

\section{Introduction}

Supersymmetry (SUSY) is one of the firmest candidates for physics beyond the Standard Model (SM) of particle interactions. If new particles are discovered at the LHC, the forthcoming step will be to establish their nature. This step could be performed at a high energy $e^{+} e^{-}$linear collider. One of the basic predictions of SUSY is the equality of the couplings in the different sectors: the SM gauge couplings must be equal to the superpartners gauge couplings and to the Yukawa coupling between a particle and its superpartner.

We present a full one-loop computation of the electroweak (EW) corrections to the sfermion partial decay width

$\Gamma\left(\tilde{f} \rightarrow f^{\prime} \chi\right)$

$\tilde{f}$ being a sfermion, $f^{\prime}$ a SM fermion and $\chi$ a chargino $\left(\chi_{1,2}^{ \pm}\right)$or a neutralino $\left(\chi_{1 \ldots 4}^{0}\right)$. The process (11) probes the fermion-sfermiongaugino/higgsino coupling, and can be used to test the SUSY relations. As we will see, the ra-

\footnotetext{
*Talk presented at 6th International Symposium on Radiative Corrections Application of Quantum Field Theory to Phenomenology (RADCOR 2002) and 6th Zeuthen Workshop on Elementary Particle Theory Loops and Legs in Quantum Field Theory, Kloster Banz, Germany, 8-13 September, 2002.
}

diative corrections induce finite shifts in these relations which are non-decoupling.

The QCD corrections to the process (1), in the framework of the Minimal Supersymmetric Standard Model (MSSM), were computed in [ 1]. However, the electroweak effects are much more cumbersome as their computation requires the renormalization of the whole MSSM Lagrangian. A first estimate was given in [2], where the contribution from the Yukawa couplings of bottom quarks decaying into chargino-neutralinos was analyzed within the so-called higgsino approximation. The electroweak effects have been further elaborated in [3].

\section{Renormalization and radiative correc- tions}

The computation to one-loop level of the partial decay width (1) requires the renormalization of the full MSSM Lagrangian, taking into account the relations among the different sectors and the mixing parameters. We choose to work in an on-shell renormalization scheme, in which the renormalized parameters are the measured quantities. The SM sector is renormalized according to the standard on-shell SM $\alpha$-scheme [ \#], and the MSSM Higgs sector (in particular the renor- 
malization of $\tan \beta$ ) is treated as in [ [5].

As far as the sfermion sector is concerned, each squark doublet contains 5 independent parameters. We choose to use the following set of input parameters?:

$\left(m_{\tilde{b}_{1}}, m_{\tilde{b}_{2}}, \theta_{b}, m_{\tilde{t}_{2}}, \theta_{t}\right), \quad m_{\tilde{f}_{1}}>m_{\tilde{f}_{2}}$.

The remaining parameters are computed as a function of those in (2). In particular, the trilinear soft-SUSY-breaking couplings read:

$A_{\{b, t\}}=\mu\{\tan \beta, \cot \beta\}+\frac{m_{\tilde{f}_{1}}^{2}-m_{\tilde{f}_{2}}^{2}}{2 m_{f}} \sin 2 \theta_{f}$,

with $\tan \beta=v_{2} / v_{1}$, the ratio of the vacuum expectation values of the two Higgs boson doublets. The mass parameters in (2) are defined to be onshell. For the mixing angle counterterm we adopt the following convention:

$\delta \theta_{f}=\frac{1}{2} \frac{\Sigma_{\tilde{f}}^{21}\left(m_{\tilde{f}_{2}}^{2}\right)+\Sigma_{\tilde{f}}^{12}\left(m_{\tilde{f}_{1}}^{2}\right)}{m_{\tilde{f}_{2}}^{2}-m_{\tilde{f}_{1}}^{2}}$,

being $\Sigma^{i j}\left(k^{2}\right)$ the mixing self-energy between sfermions $i$ and $j$. The convention (4) is known to introduce gauge parameter dependence in the definition of the mixing angle, but the result is the same as the gauge independent computation of the self-energies in (4i) within the 't HooftFeynman gauge [ 6]. Since the heaviest topsquark mass is not an input parameter, it receives finite radiative corrections:

$\Delta m_{\tilde{t}_{1}}^{2}=\delta m_{\tilde{t}_{1}}^{2}+\Sigma_{\tilde{t}_{1}}\left(m_{\tilde{t}_{1}}^{2}\right)$,

where $\delta m_{\tilde{t}_{1}}^{2}$ is a combination of the counterterms of the parameters in (2), and the counterterms of the gauge and Higgs sectors.

The chargino/neutralino sector contains six particles, but only three independent input parameters $\left(M\right.$ and $M^{\prime}$-the soft-SUSY-breaking $S U(2)_{L}$ and $U(1)_{Y}$ gaugino masses- and the higgsino mass parameter $\mu$ ). The situation in this sector is quite different from the sfermion case, since in this case no independent counterterms for the mixing matrix elements can be introduced. We stick to the following procedure: First,

${ }^{2}$ Throughout this work we make use of third generation notation. The notation is as in [2, 3]. we introduce a set of renormalized parameters $\left(M, M^{\prime}, \mu\right)$ in the expression of the chargino and neutralino matrices $\left(\mathcal{M}\right.$ and $\left.\mathcal{M}^{0}\right)$, and diagonalize them by means of unitary matrices $M_{D}=$ $U^{*} \mathcal{M} V^{\dagger}, M_{D}^{0}=N^{*} \mathcal{M}^{0} N^{\dagger}$. Now $U, V$ and $N$ must be regarded as renormalized mixing matrices. The counterterm mass matrices are then $\delta M_{D}=U^{*} \delta \mathcal{M} V^{\dagger}, \delta M_{D}^{0}=N^{*} \delta \mathcal{M}^{0} N^{\dagger}$, which are non-diagonal. At this point, we introduce renormalization conditions for certain elements of $\delta M_{D}$ and $\delta M_{D}^{0}$. In particular, using the unitarity properties of $U$ and $V$ we can write the following set of equations

$$
\begin{gathered}
M_{1} \delta M_{1}+M_{2} \delta M_{2}=M \delta M+\mu \delta \mu+\delta M_{W}^{2} \\
M_{1} M_{2}\left(M_{1} \delta M_{2}+M_{2} \delta M_{1}\right)= \\
=\left(M \mu-M_{W}^{2} s_{2 \beta}\right) \times[M \delta \mu+\mu \delta M \\
\left.-M_{W}^{2} \delta s_{2 \beta}-s_{2 \beta} \delta M_{W}^{2}\right] .
\end{gathered}
$$

$M_{1}$ and $M_{2}$ are the chargino on-shell masses, and $\delta M_{i}$ is a shortcut for the following combination of chargino self-energies:

$\frac{\delta M_{i}}{M_{i}}=-\frac{1}{2}\left(\Sigma_{L}^{-i}\left(M_{i}^{2}\right)+\Sigma_{R}^{-i}\left(M_{i}^{2}\right)\right)-\Sigma_{S}^{-i}\left(M_{i}^{2}\right)$

Conditions (6) and (7) are equivalent to one-loop on-shell renormalization of the chargino masses. As a last renormalization condition, we require on-shell renormalization for one of the neutralinos, which we choose the lightest one, and find the expression for the $M^{\prime}$ counterterm:

$\delta M^{\prime}=\frac{1}{N_{11}^{* 2}}\left(\delta M_{1}^{0}-\sum_{\alpha \text { or } \beta \neq 1} N_{1 \alpha}^{*} \delta \mathcal{M}_{\alpha \beta}^{0} N_{1 \beta}^{*}\right)$,

where $\delta M_{1}^{0}$ is as in (7), but for te lightest neutralino $\left(\chi_{1}^{0}\right)$. Solving eqs. (6) for $\delta M$ and $\delta \mu$, and using eq. (8) for $\delta M^{\prime}$, the renormalization conditions for the chargino/neutralino sector are complete. The other neutralino masses receive radiative corrections. In this framework the renormalized one-loop chargino/neutralino 2-point functions are non-diagonal. Therefore one must take into account this mixing either by including explicitly the reducible $\chi_{r}-\chi_{s}$ mixing diagrams, or by means of external mixing wave-function terms, e.g. the left- and right-chiral form factors for the 
creation of a neutralino $\chi_{\alpha}^{0}$ must be multiplied by,

$$
\begin{aligned}
& \mathcal{Z}_{R}^{0 \beta \alpha}=\frac{1}{M_{\alpha}^{0^{2}}-M_{\beta}^{0^{2}}} \times \\
& \quad\left[M_{\beta}^{0} \hat{\Sigma}_{S L}^{\beta \alpha}\left(M_{\alpha}^{0^{2}}\right)+M_{\alpha}^{0} \hat{\Sigma}_{S R}^{\beta \alpha}\left(M_{\alpha}^{0^{2}}\right)\right. \\
& \left.\quad+M_{\beta}^{0} M_{\alpha}^{0} \hat{\Sigma}_{L}^{\beta \alpha}\left(M_{\alpha}^{0^{2}}\right)+M_{\alpha}^{0^{2}} \hat{\Sigma}_{R}^{\beta \alpha}\left(M_{\alpha}^{0^{2}}\right)\right] \\
& \mathcal{Z}_{L}^{0 \beta \alpha}=\frac{1}{M_{\alpha}^{0^{2}-M_{\beta}^{02}} \times} \\
& \quad\left[M_{\beta}^{0} \hat{\Sigma}_{S R}^{\beta \alpha}\left(M_{\alpha}^{0^{2}}\right)+M_{\alpha}^{0} \hat{\Sigma}_{S L}^{\beta \alpha}\left(M_{\alpha}^{0^{2}}\right)\right. \\
& \left.\quad+M_{\beta}^{0} M_{\alpha}^{0} \hat{\Sigma}_{R}^{\beta \alpha}\left(M_{\alpha}^{0^{2}}\right)+M_{\alpha}^{0^{2}} \hat{\Sigma}_{L}^{\beta \alpha}\left(M_{\alpha}^{0^{2}}\right)\right]
\end{aligned}
$$

Here $\hat{\Sigma}_{*}^{\beta \alpha}\left(k^{2}\right)$ are the renormalized mixing twopoint functions $\chi_{\beta}^{0} \rightarrow \chi_{\alpha}^{0}$. Equivalent expressions can be worked out for the chargino sector. See Refs. [7] for different (but one-loop equivalent) approaches to the renormalization of the chargino/neutralino sector.

The complete one-loop computation consists of:

- renormalization constants for the parameters and wave functions in the bare Lagrangian,

- one-loop one-particle irreducible three-point functions,

- mixing terms among the external charginos and neutralinos,

- soft- and hard- photon bremsstrahlung.

All kind of MSSM particles are taken into account in the loops: SM fermions, sfermions, electroweak gauge bosons, Higgs bosons, Goldstone bosons, Fadeev-Popov ghosts, charginos, neutralinos. The computation is performed in the 't Hooft-Feynman gauge, using dimensional reduction for the regularization of divergent integrals. The loop computation itself is done using the computer algebra packages FeynArts 3.0 and FormCalc 2.2 [ 8, 9]. The numerical evaluation of one-loop integrals makes use of Loop Tools 1.2 [ 9].

\section{Results}

The results show the very interesting property that none of the particles of the MSSM decouples from the corrections to the observables (11). This can be well understood in terms of renormalization group (RG) running of the parameters and SUSY breaking. Take, e.g., the effects

\footnotetext{
${ }^{3}$ The resulting FORTRAN code can be obtained from http://www-itp.physik. uni-karlsruhe.de/ guasch/progs/.
}

of squarks in the electron-selectron-photino coupling. Above the squark mass scale $\left(Q>m_{\tilde{q}}\right)$ the electron electromagnetic coupling $(\alpha(Q))$ is equal (by SUSY) to the electron-selectron-photino coupling $(\tilde{\alpha}(Q))$, and both couplings run according to the same $\mathrm{RG}$ equations. At $Q=m_{\tilde{q}}$ the squarks decouple from the $\mathrm{RG}$ running of the couplings. At $Q<m_{\tilde{q}}, \alpha(Q)$ runs due to the contributions from pure quark loops, but $\tilde{\alpha}(Q)$ does not run anymore, and it is frozen at the squark scale, that is: $\tilde{\alpha}\left(Q<m_{\tilde{q}}\right)=\alpha\left(m_{\tilde{q}}\right)$. Therefore, when comparing these two couplings at a scale $Q<m_{\tilde{q}}$, they differ by the logarithmic running of $\alpha(Q)$ from the squark scale to $Q$ : $\tilde{\alpha}(Q) / \alpha(Q)-1=\beta \log \left(m_{\tilde{q}} / Q\right)$.

The above discussion has two important consequences:

1) The non-decoupling can be used to extract information of the high-energy part of the SUSY spectrum: one can envisage a SUSY model in which a significant splitting among the different SUSY masses exists, e.g. $m_{\tilde{q}} \gg m_{\tilde{l}}$, where the sleptons lie below the production threshold in an $e^{+} e^{-}$linear collider, but the squarks are above it. By means of high precision measurements of the lepton-slepton-chargino/neutralino couplings one might be able to extract information of the squark sector of the model, to be checked with the available data from the LHC.

2) By the same token, it means that the value of the radiative corrections depends on all parameters of the model, and we can not make precise quantitative statements unless the full SUSY spectrum is known. This drawback can be partially overcome by the introduction of effective coupling matrices, which can be defined as follows. The subset of fermion-sfermion one-loop contributions to the self-energies of gauge-boson, Higgs-bosons, Goldstone-bosons, charginos and neutralinos form a gauge invariant finite subset of the corrections. Therefore these contributions can be absorbed into a finite shift of the chargino/neutralino mixing matrices $U, V$ and $N$ appearing in the couplings: $U^{\text {eff }}=U+$ $\Delta U^{(f)}, V^{e f f}=V+\Delta V^{(f)}, N^{e f f}=N+\Delta N^{(f)}$. In this way we can decouple the computation of the universal (or super-oblique [10]) corrections. These corrections contain the non-decoupling log- 
arithms from sfermion masses. We have been able to derive an analytic expression containing these logarithms in a simple case. We have computed the electron-selectron contributions to $\Delta U^{(f)}$ and $\Delta V^{(f)}$ matrices, assuming zero mixing angle in the selectron sector $\left(\theta_{e}=0\right)$, we have identified the leading terms in the approximation $m_{\tilde{e}_{i}}, m_{\tilde{\nu}} \gg\left(M_{W}, M_{i}\right) \gg m_{e}$, and analytically cancelled the divergences and the renormalization scale dependent terms; finally, we have kept only the terms logarithmic in the slepton masses. The result for $\Delta U^{(f)}$ reads as follows:

$$
\begin{aligned}
& \Delta U_{i 1}^{(f)}=\frac{\alpha}{4 \pi s_{W}^{2}} \log \left(\frac{M_{\tilde{e}_{L}}^{2}}{M_{X}^{2}}\right)\left[\frac{U_{i 1}^{3}}{6}-\right. \\
& \quad U_{i 2} \frac{\sqrt{2} M_{W}\left(M c_{\beta}+\mu s_{\beta}\right)}{3\left(M^{2}-\mu^{2}\right)\left(M_{1}^{2}-M_{2}^{2}\right)^{2}}\left(M^{4}-M^{2} \mu^{2}+\right. \\
& \quad+3 M^{2} M_{W}^{2}+\mu^{2} M_{W}^{2}+M_{W}^{4}+M_{W}^{4} c_{4 \beta} \\
& \left.\left.\quad+\left(\mu^{2}-M^{2}\right) M_{i}^{2}+4 M \mu M_{W}^{2} s_{2 \beta}\right)\right] \\
& \Delta U_{i 2}^{(f)}=\frac{\alpha}{4 \pi s_{W}^{2}} \log \left(\frac{M_{\tilde{e}_{L}}^{2}}{M_{X}^{2}}\right) U_{i 1} \times \\
& \quad \times \frac{M_{W}\left(M c_{\beta}+\mu s_{\beta}\right)}{3 \sqrt{2}\left(M^{2}-\mu^{2}\right)\left(M_{1}^{2}-M_{2}^{2}\right)^{2}}\left(\left(M^{2}-\mu^{2}\right)^{2}+\right. \\
& \quad+4 M^{2} M_{W}^{2}+4 \mu^{2} M_{W}^{2}+2 M_{W}^{4}+ \\
& \left.\quad+2 M_{W}^{4} c_{4 \beta}+8 M \mu M_{W}^{2} s_{2 \beta}\right)
\end{aligned}
$$

$M_{\tilde{e}_{L}}^{2}$ being the soft-SUSY-breaking mass of the $\left(\tilde{e}_{L}, \tilde{\nu}\right)$ doublet, whereas $M_{X}$ is a SM mass. In the on-shell scheme for the SM electroweak theory we define parameters at very different scales, basically $M_{X}=M_{W}$ and $M_{X}=m_{e}$. These wideranging scales enter the structure of the counterterms and so must appear in eq.(10) too. As a result the leading $\log$ in the various terms of this equation will vary accordingly. For simplicity in the notation we have factorized $\log M_{\tilde{e}_{L}}^{2} / M_{X}^{2}$ as an overall factor. In some cases this factor can be very big, $\log M_{\tilde{e}_{L}}^{2} / m_{e}^{2}$; it comes from the electronselectron contribution to the chargino-neutralino self-energies.

Although above we have singled out the nondecoupling properties of sfermions, we would like to stress that the whole spectrum shows nondecoupling properties. By numerical analysis we have been able to show the existence of logarithms of the gaugino mass parameters $\left(M / M_{X}\right.$ and $\left.M^{\prime} / M_{X}\right)$, and the Higgs mass $\left(M_{H^{ \pm}} / M_{X}\right)$. However, due to the complicated mixing structure of

\begin{tabular}{|c|c|c|c|c||c|}
\hline & $\chi_{1}^{0}$ & $\chi_{2}^{0}$ & $\chi_{3}^{0}$ & $\chi_{4}^{0}$ & $\chi_{1}^{+}$ \\
\hline \multicolumn{7}{|c|}{$B R\left(\tilde{b}_{1} \rightarrow q \chi\right)$} \\
\hline Tree & 0.272 & 0.092 & 0.047 & 0.014 & 0.575 \\
\hline QCD & 0.308 & 0.104 & 0.031 & 0.018 & 0.538 \\
\hline Total & 0.291 & 0.092 & 0.031 & 0.018 & 0.568 \\
\hline \hline \multicolumn{7}{|c|}{$B R\left(\tilde{b}_{2} \rightarrow q \chi\right)$} \\
\hline Tree & 0.502 & 0.332 & 0.123 & - & 0.042 \\
\hline QCD & 0.541 & 0.386 & 0.054 & - & 0.019 \\
\hline Total & 0.528 & 0.395 & 0.056 & - & 0.020 \\
\hline
\end{tabular}

Table 1

Branching ratios of bottom-squarks into charginos and neutralinos for the parameter set (11). Shown are: the tree-level value, the QCD-corrected value, and the value including EW corrections. Branching ratios below $10^{-3}$ are not shown.

the model, we were not able to derive simple analytic expressions containing these non-decoupling logarithms. Note that in any observable which includes the fermion-sfermion-chargino/neutralino Yukawa couplings at leading order we will have this kind of corrections, therefore the full MSSM spectrum must be taken into account when computing radiative corrections, since otherwise one could be missing large logarithmic contributions of the heavy masses.

As for the non-universal part of the contributions, we summarize here the main features:

a) The corrections grow as the logarithm squared of the decaying particle mass, due to the presence of electroweak Sudakov double-logs [11].

b) The corrections show multiple threshold singularities when varying any of the parameters of the model, however they are well behaved in the regions between thresholds. Therefore, in order to give a quantitative value of the corrections the correlation between the different particle masses must be known.

c) The third sfermion family contains a large contribution that can be traced back to the presence of corrections similar to the threshold-like corrections to the bottom-quark (and $\tau$-lepton) Yukawa coupling $\Delta m_{\{b, \tau\}}$ [ 12]. This contribution contains (aside from $\Delta m_{\{b, \tau\}}$ ) terms from the sfermion mixing self-energies $\tilde{f}_{L}-\tilde{f}_{R}$, and 
is (approximately) proportional to the sfermion mass splitting. It is also present in the QCD corrections.

d) The three-point vertex functions contain the soft SUSY-breaking trilinear Higgs-sfermionsfermion couplings $A_{f}$. If $A_{f}$ is large, it would induce large corrections (even larger than 100\%!). However, as long as $A_{f}$ is in the perturbative coupling regime, the corrections stay below $20 \%$.

Points c) and d) play a complementary role in the large $\tan \beta$ regime due to the relation (3). A small mass splitting (or small mixing angle) means small $\Delta m_{\{b, \tau\}}$ corrections, but a large value of $A_{\{b, \tau\}} \simeq \mu \tan \beta$. However a large $A_{f}$ value would both spoil perturbativity and generate charge and/or colour breaking vacua. Therefore, at large $\tan \beta$ the bottom-squarks and $\tau$ sleptons must have significant splitting and mixing angle, providing large corrections of the type c) above.

The $\Delta m_{\{b, \tau\}}$ corrections play also an important role in the phenomenology of the MSSM Higgs bosons at large $\tan \beta$, showing nondecoupling properties for $M_{S U S Y} \gg M_{S M}, M_{H^{ \pm}}$, as stressed e.g. in [13]. The $\tilde{f}_{a}-f^{\prime}-\chi_{r}$ coupling, on the other hand, exhibits a new kind of nondecoupling properties, which are independent of the value of $\tan \beta$.

As an example of the numerical value of the corrections, we present in Table 11 the treelevel and corrected branching ratios for bottomsquarks, for the following set of input parameters:

$$
\begin{aligned}
& m_{t}=175 \mathrm{GeV}, m_{b}=5 \mathrm{GeV}, \tan \beta=4, \\
& m_{\tilde{b}_{2}}=m_{\tilde{d}_{2}}=m_{\tilde{u}_{2}}=m_{\tilde{e}_{2}}=300 \mathrm{GeV}, \\
& m_{\tilde{b}_{1}}=m_{\tilde{d}_{1}}=m_{\tilde{e}_{1}}=m_{\tilde{b}_{2}}+5 \mathrm{GeV} \\
& m_{\tilde{u}_{2}}=290 \mathrm{GeV}, m_{\tilde{t}_{2}}=300 \mathrm{GeV} \\
& \theta_{b}=\theta_{d}=\theta_{u}=\theta_{e}=0, \theta_{t}=-\pi / 5 \\
& \mu=150 \mathrm{GeV}, M=250 \mathrm{GeV}, \\
& M_{H^{ \pm}}=120 \mathrm{GeV}, m_{\tilde{g}}=500 \mathrm{GeV} .
\end{aligned}
$$

The results of Table 1 show that a high precision measurement of the partial decay branching ratios of sfermions, to be performed at a high energy $e^{+} e^{-}$linear collider, is sensitive to the EW corrections. From Table 1 it is also clear that the EW corrections can induce a change on the branching ratios of the leading decay channels comparable to the QCD corrections. Therefore both contribu- tions must be taken into account on equal footing in the analysis of the phenomenology of sfermions.

Acknowledgments: The calculations have been done using the QCM cluster of the DFG Forschergruppe "Quantenfeldtheorie, Computeralgebra und Monte-Carlo Simulation". This collaboration is part of the network "Physics at Colliders" of the European Union under contract HPRNCT-2000-00149. The work of J.G. has been partially supported by the European Union under contract No. HPMF-CT-1999-00150. The work of J.S. has been supported in part by MECYT and FEDER under project FPA2001-3598.

\section{REFERENCES}

1. S. Kraml et al., Phys. Lett. B386 (1996) 175; A. Djouadi, W. Hollik, C. Jünger, Phys. Rev. D55 (1997) 6975; W. Beenakker et al., Z. Phys. C75 (1997) 349.

2. J. Guasch, W. Hollik, J. Solà, Phys. Lett. B437 (1998) 88.

3. J. Guasch, W. Hollik, J. Solà, Phys. Lett. B510 (2001) 211; hep-ph/0207364.

4. M. Böhm, H. Spiesberger, W. Hollik, Fortsch. Phys. 34 (1986) 687; W. Hollik, Fortschr. Phys. 38 (1990) 165.

5. P. Chankowski, S. Pokorski, J. Rosiek, Nucl. Phys. B423 (1994) 437; A. Dabelstein, Z. Phys. C67 (1995) 495; Nucl. Phys. B 456 (1995) 25.

6. J. R. Espinosa, Y. Yamada, hep-ph/0207351.

7. H. Eberl, et al., Phys. Rev. D64 (2001) 115013; T. Fritzsche, W. Hollik, Eur. Phys. J. C24 (2002) 619.

8. T. Hahn, Comput. Phys. Commun. 140 (2001) 418; T. Hahn, C. Schappacher, Comput. Phys. Commun. 143 (2002) 54.

9. T. Hahn, M. Pérez-Victoria, Comput. Phys. Commun. 118 (1999) 153; T. Hahn, FeynArts, Form Calc and Loop Tools user's guides, available from http: //www . feynarts.de;

10. E. Katz, L. Randall, S. Su, Nucl. Phys. B536 (1998) 3 .

11. P. Ciafaloni, D. Comelli, Phys. Lett. B446 (1999) 278; V. S. Fadin, et al., Phys. Rev. D61 (2000) 094002; M. Beccaria, et al., Phys. 
Rev. D65 (2002) 093007.

12. L.J. Hall, R. Rattazzi, U. Sarid, Phys. Rev. D50 (1994) 7048; M. Carena, et al., Nucl. Phys. B426 (1994) 269.

13. J.A. Coarasa et al. Eur. Phys. J. C2 (1998) 373; M. Carena et al., Nucl. Phys. B577 (2000) 88; A. Belyaev, J. Guasch, J. Solà, these proceedings. 